\title{
Distributed medium access control with conditionally altruistic users
}

\author{
Panayotis Antoniadis ${ }^{*}$, Serge Fdida ${ }^{2}$, Christopher Griffin ${ }^{3}$, Youngmi Jin ${ }^{4}$ and George Kesidis ${ }^{3}$
}

\begin{abstract}
In this paper, we consider medium access control of local area networks (LANs) under limited-information conditions as befits a distributed system. Rather than assuming 'by rule' conformance to a protocol designed to regulate packet-flow rates (e.g., CSMA windowing), we begin with a non-cooperative game framework and build a dynamic, conditional, altruism term into the net utility. The effects of altruism are analyzed at Nash equilibrium for the carrier sense multiple access (CSMA)-like random-access framework in the quasi-stationary (fictitious play) regime. We consider either power or throughput-based costs of networking, and the cases of identical or heterogeneous (independent) players. Unlike related work in this area, our objective is not to optimize the performance of the system but to formulate a realistic economic model capturing altruistic motivations. This model can then form the basis for higher-level incentive schemes that will encourage altruistic, instead of selfish, behavior. In a numerical study we consider the cases of identical or diverse players and show interesting relationships between the main parameters of our model and comparisons with other alternatives discussed in the literature.
\end{abstract}

Keywords: Medium access control; Resource allocation; Altruism; Game theory

\section{Introduction}

Flow and congestion control are fundamental networking problems due to the distributed, information-limited nature of the decision-making process in many popular access technologies. Various distributed mechanisms have been implemented to cooperatively desynchronize demand, e.g., Transmission Control Protocol (TCP), ALOHA, and carrier sense multiple access (CSMA). Typically, when congestion is detected, all end-devices are expected to slow down their transmission rates and then slowly increase again hoping to find a fair and efficient equilibrium.

If some users $\mathrm{a}^{\mathrm{a}}$ employ alternative implementations of the prescribed 'by rule' protocols, e.g., ones that slow down less than they should or even increase their transmission rate in the presence of congestion, the result could be an unfair allocation or even congestion collapse (see e.g., [1,2]). The experience with TCP (e.g., [3]) has shown that developers do create versions of the protocol that depart from the standard cooperative (by rule)

*Correspondence: antoniadis@tik.ee.ethz.ch

${ }^{1}$ ETH Zurich, Zurich, Switzerland

Full list of author information is available at the end of the article congestion-avoidance algorithm, like Turbo TCP. Outside of a tactical context, non-cooperative tactics at the medium access control (MAC) level have not been as widespread, perhaps due to the increased difficulty of modifying lower-level networking drivers by users or third parties, but such modifications are possible. As more network users behave selfishly and thereby more significantly reduce the performance of the rest, the other players are increasingly incentivized to adopt selfish strategies themselves, potentially leading to deadlock.

To address this threat, there is a steadily growing literature that analyzes the equilibria of different distributed network resource allocation games [1,4-13]. Such models provide useful insights on the expected equilibria when users do have the option to choose alternative implementations of the MAC protocol and constitute a framework for devising and analyzing incentive mechanisms to encourage the behavior that would lead to the most desirable equilibria. For example, in a Markovian setting without fictitious play, ${ }^{\mathrm{b}} \mathrm{Ma}$ et al. [13] introduce a cooperation parameter (a probability to stop transmitting) and then follows a detection and punishment methodology regarding selfish behavior.

\section{Springer}

(C) 2013 Antoniadis et al.; licensee Springer. This is an Open Access article distributed under the terms of the Creative Commons Attribution License (http://creativecommons.org/licenses/by/2.0), which permits unrestricted use, distribution, and reproduction in any medium, provided the original work is properly cited. 
In addition, even when users do follow the prescribed protocol, game theoretical models could be used as analytical frameworks that enable more informed choices in the implementation of the corresponding flow and congestion control protocols (e.g., by associating a utility function to end-devices, which can then be the basis of actions by rationally selfish players). To this end, for a random-access local area network (LAN), several authors have recently considered the problem of distributed optimization of a global objective (total throughput, social welfare) subject to a fairness constraint. For example, in [12], a utility function design problem is studied considering estimation errors of the network state.

Our work falls into the former category of gametheoretic models but is different than the typical approach in addressing potentially selfish behavior at the MAC layer. Our objective is to formulate a more realistic utility model that captures altruistic motivations. As discussed in the next section, research in the field of experimental economics has demonstrated that such motivations do play an important role in a wide variety of public goods and common-pool games. Our analysis could then form the basis for advanced incentive schemes, which, instead of attempting to punish selfish behavior, would aim to encourage altruistic behavior under certain conditions. For example, a possible realistic outcome could be the design of a high-level user interface which will allow users to set the urgency of their communication and which encourages users to assign lower priority to their traffic when there is evidence that other users generally are doing the same, $c f$. Section 7. If successful, such a mechanism will not only improve performance at any given moment but it will also allow certain users to increase their own throughput only when they really need it, improving this way also the efficiency of the system over time without the need for complex and unattractive pricing schemes (e.g., [4]).

In the long term we have to deal with an 'evolutionary' game, as defined in [14], that could pass from different states, as in [15]: In one state, all participants follow a basically cooperative strategy (cooperative by rule protocol), though the context here is an information-limited distributed system. From this cooperative state, suppose that some players defect from the cooperative protocol and thereby (perhaps 'greedily') achieve better performance for themselves at the expense of all other players. A sufficient number of defectors (i.e., sufficiently poor performance for non-defectors) will incentivize all remaining players to defect, thus possibly leading to a 'fully noncooperative' state. In the fully non-cooperative state, an information-limited game may be prone to non-Pareto (even deadlocked) equilibria. One approach to this problem is to employ mechanisms to overcome 'loss aversion'
[16] and thereby explore play actions that may yield suboptimal net utilities in the short term (i.e., moves that appear locally irrational) but avoid getting trapped in non-Pareto equilibria in the longer term. Alternatively again from the fully non-cooperative state, the possibility for users to lower the priority of their transmission based on information about the behavior of others can result in a conditionally cooperative state that could help escape from the deadlock and possibly lead back to the fully cooperative one. Notwithstanding such measures, the non-cooperative state may not reach the social welfare achieved in the original cooperative state, $c f$. the numerical results in Section 5.4.

As our main contribution here, we focus on the conditionally cooperative state of the system and formulate and analyze a novel CSMA control game with conditionally altruistic players. We model this situation by altering the net utilities of the players with a term which we identify with altruism. Altruistic tactics in evolutionary/meanfield games have long been considered; see [17] as a recent reference. In networking, altruism has been modeled as a user's statically personalized weight on the utility of others in games of network formation [18], packet forwarding in delay tolerant networks [19], routing [20,21], and medium access control by us in [22].

However, we argue in detail in the next section that such static altruistic models, although theoretically interesting, fail to capture important realistic attributes of altruistic behavior studied in the behavioral and experimental economics literature. In this paper, we formulate a fictitious play model where altruism by one user is based on the perceived mean modulated throughput of the other players ${ }^{\mathrm{c}}$ (i.e., made 'dynamic') by factoring the estimated mean total channel idle time. Unlike Heusse et al. [23], who propose a window-update algorithm that tries to directly minimize the average idle time of the channel, in our model users will use less than their 'fair' share when they do not really need it, but under the constraint that others do the same. For example, large idle time may be a signal that competing devices are also behaving in a socially sensitive manner, expressing a cooperative 'social norm.' In this case, excessive altruism may result in an underused channel. We should stress that our objective is not to optimize the overall throughput of the system but to study the stable equilibria that such altruistic devices could reach. Finally, we do not assume that the users share information and act in a coordinated fashion, i.e., so as to form a player coalition.

This paper is outlined as follows. In Section 2, we give a brief background on altruistic behavior. A fictitious play model with dynamic altruism for a slotted ALOHA LAN is given in Section 3. In Section 4, some closely related variations of the LAN model are considered. Numerical studies are given in Sections 5 and 6, including for the case of 
player diversity. Finally, in Section 7, we conclude with a summary and discussion of future work.

\section{Background on altruistic behavior}

Economists are often criticized for the common assumption that humans are rational (i.e., purely self-interested), which leads to a pessimistic view of the outcome of various formulated game-theoretic models. In reality, many people act 'altruistically', defined as an 'unselfish concern for or devotion to the welfare of others.'

In fact, despite this selfishness stereotype, certain branches of economics, such as behavioral and experimental economics, do incorporate social, cognitive, and psychological factors in their models of human behavior (see [16] for a historical overview), in a way not typically captured in cooperative game-theoretic frameworks.

Two common scenarios in which altruistic behavior consistently appears in experiments with real users include the public-goods provision and common-pool resource sharing games. For example, in the traditional public-good provision game, where players determine their individual contribution toward the construction of a pure public good, experiments have challenged the assumption that free riding is always the dominant strategy (e.g., [24]). Similarly altruistic behaviors have been observed in a very simple resource-sharing game, called ultimatum, where one player decides how to share a fixed amount of money with another player who can decide whether to accept or reject sharing: here rejection leaves both with zero profit. Experiments show that people altruistically sacrifice their own profit to punish unfair decisions by others; see [25] for an overview of experiments with different variations of this game and interesting regularities observed.

An important lesson of experimental economics is that altruism does not seem to be a static and hardwired characteristic of humans but depends on many aspects of the environment. In other words, the level of altruism of an individual is dynamic and could change over time depending on the context and the behavior of the group $[25,26]$. Indeed, the cooperation rate in many experiments has been proven to be much higher if subjects know that there is a possibility of meeting the same partners again in future periods [27], when their perception on the overall level of altruism in their group is high [28], or even just by a positive framing of the experiment [29].

From these and many other contextual factors that can affect the cooperation levels in a group, social norms are perhaps the most influential (see [30,31]) but complex to incorporate in a simple economic model. To this end, Fehr and Schmidt [32] have proposed a utility function to model the altruistic behavior of people in ultimatum experiments, which incorporates a measure of fairness (or 'inequity aversion') in a static way, i.e., its main parameters are indifferent to the dynamics of the system. As a more realistic but less tractable alternative, $\mathrm{H}$. Margolis argues in favor of a more dynamic and complex model, called 'neither selfish nor exploited' [33], which proposes a dual utility model which takes into account the history of one's actions, the current overall behavior, the effect of altruistic action, and the developed norms in a society.

\section{Slotted ALOHA random-access LAN with dynamic altruism}

\subsection{Altruistic framework with power-based cost and concave utility of throughput}

In our scenario, the high complexity of human nature and the surrounding social environment plays a less important role since the cooperation game that we study is limited in time, the identity of the players are hidden, the stakes are relatively low, and the decisions of users are mediated through a programmed device.

So we propose to incorporate in a simple utility function the effect of the external manifestation of altruistic behavior that is a statistical norm as termed in [33] or simply 'what others do' [28]. To perceive this, the availability of reliable information about the group's statistical behavior is critical. Our use of the mean idle time per active player to determine the level of altruism in the system is realistic in terms of information availability since it can be easily measured by the different users; though, again, low demand could be mistakenly taken for altruistic behavior and congestion due to a high number of competing users could be mistaken as individually selfish behavior (see discussion on the results shown in Table 1).

Consider a slotted ALOHA random access LAN wherein the $N \geq 2$ participating nodes control their access probability parameter, $q$. A basic assumption is that nodes' control actions are based on observations in steady state, i.e., 'fictitious play' [34], resulting in a quasistationary dynamical system $[4,6,35]$ based on the mean throughputs, i.e., for player $i$ :

$$
\gamma_{i}(\underline{q})=q_{i} \prod_{j \neq i}\left(1-q_{j}\right) .
$$

Another basic assumption in the following is that the source of a successful transmission is evident to all other participating nodes. We further assume that the degree of

\section{Table 1 Chicken game}

P2

\begin{tabular}{lcc} 
P1 & Tx & No Tx \\
\hline Tx & $(-\xi,-\xi)$ & $(0,1-\xi)$ \\
no Tx & $(1-\xi, 0)$ & $(0,0)$ \\
\hline
\end{tabular}

The net payoffs for collective action (transmit (Tx) or not) by the players (P1,P2) in the chicken game. 
altruism $\alpha_{i}$ of each node $i$ depends on the activity of the other users as

$$
\begin{aligned}
\alpha_{i}\left(\underline{q}_{-i}\right) & =\prod_{j \neq i}\left(1-q_{j}\right)=\frac{\gamma_{i}(\underline{q})}{q_{i}} \\
& =\gamma_{i}(\underline{q})+\prod_{j}\left(1-q_{j}\right),
\end{aligned}
$$

where the second term is just the mean idle time of the channel; thus, every node can easily estimate its (dynamic) altruism. By using its control action (strategy), $q_{i}$, each $i$ seeks to maximize its net utility:

$$
V_{i}(\underline{q})=C_{i} \log \left(\gamma_{i}(\underline{q})\right)+A_{i} \alpha_{i}\left(\underline{q}_{-i}\right) \bar{\gamma}_{-i}(\underline{q})-M_{i} q_{i}
$$

where the dynamic altruism factor $\alpha$ modulates the contribution of the mean service of all other players to the net utility of player $i$,

$$
\bar{\gamma}_{-i}(\underline{q})=\frac{1}{N-1} \sum_{j \neq i} \gamma_{j}(\underline{q})
$$

the utility derived by one's own throughput is modulated by a concave function $[4,7,35]$ as modeled here in the form of a logarithm (for tractability); and we have assumed a power-based $\operatorname{cost}^{\mathrm{e}} M q$. Note that because we assume that the source of each successfully transmitted packet is evident to all nodes, each node $i$ can easily estimate $\bar{\gamma}_{-i}$. Again, though each player $i$ optimizes $V_{i}$ in a noncooperative fashion, the game is called altruistic to reflect the second term in (1). In summary, in our model of an altruistic player $i$, benefit (utility) is derived from the success of others $\left(\bar{\gamma}_{-i}\right)$ and channel idleness $\left(\alpha_{i}\right)$, the latter indicating altruism on the part of others ${ }^{\mathrm{f}}$.

Note that in classical ALOHA, choosing very high (re)transmission parameter $q$ results in wasted slots due to interference and wasted transmission power, and choosing very low $q$ results in underused (empty) slots. Also note that a single-play slotted ALOHA game between two identical players is similar to the game chicken. If $\xi<1$ is the cost of transmission and the (normalized) payoff of successful transmission is 1 , then the Table 1 gives net payoffs for collective action (transmit (Tx) or not) by the players $(\mathrm{P} 1, \mathrm{P} 2)$.

The single-play game has three Nash equilibria: two 'pure' strategies, (Tx, no-Tx) and (no-Tx, Tx), and one mixed strategy: Tx with probability $q^{*}$ (and no Tx with probability $\left.1-q^{*}\right)$, where $q^{*}=1-\xi$ jointly minimizes the expected net gains, $(1-\xi) q_{k}\left(1-q_{3-k}\right)-\xi q_{k} q_{3-k}$, of players $k \in\{1,2\}$.

In the following, we consider an iterated version of this game where players pursue mixed strategies based on observations of throughput $\gamma_{i}$ observed in steady state.

Note that if we further assume that nodes are aware of the $C, M$ parameters of other nodes, then we can replace $\bar{\gamma}$ with the net utility of the other players as in [22] (particularly for throughput-based costs $M \gamma$ ).

Proposition 3.1. If the game is synchronous play and all users $i$ have the same (normalized) parameters

$$
c:=C_{i} / M_{i}<1 \text { and } a:=A_{i} / M_{i},
$$

then there is a symmetric Nash equilibrium $\underline{q}^{*}=q^{*} \underline{1}$, where $0<q^{*}<1$ is a solution to

$$
f(q):=a q^{2}(1-q)^{2 N-3}+q-c=0 .
$$

Proof. When $q_{i}=q$ for all $i$, the first-order necessary conditions of a symmetric Nash equilibrium,

$$
0=\frac{\partial V_{i}}{\partial q_{i}}(q \underline{1})=-\frac{M}{q} f(q),
$$

i.e., equivalent to (3). Note that $f(0)=-c<0$ and $f(1)=$ $1-c>0$, the latter by hypothesis. So, by the continuity of $f$ and the intermediate value theorem, a root of $f$ exists in $(0,1)$.

All such solutions $q^{*} \underline{1}$ correspond to Nash equilibria because $\partial^{2} V_{i}(\underline{q}) / \partial q_{i}^{2}=-C_{i} / q_{i}^{2}<0$ for all $i, \underline{q}$.

The following corollary is immediate.

Corollary 3.1. There is a unique symmetric Nash equilibrium point (NEP) if $\min _{q \in(0,1)} f^{\prime}(q)>0$ (i.e., f is strictly increasing), a condition on parameters $N$ and $a$.

Note that there may be non-symmetric Nash equilibria in these games, even for the case of homogeneous users, e.g., [36]. Also, it is well known that Nash equilibria of iterative games are not necessarily asymptotically stable, e.g., [37-39]. In [4,35] for a slotted ALOHA game with throughput-based costs $M \gamma$, using a Lyapunov function for arbitrary $N \geq 2$ players, a non-cooperative two-player ALOHA was shown to have two different interior ${ }^{\mathrm{g}}$ Nash equilibria, only one of which was locally asymptotically stable (see also [40]).

For stability analysis of our altruistic game, consider the discrete-time $(n)$, synchronous-play gradient-ascent dynamics,

$$
q_{i}(n)=\arg \max _{q_{i}} V_{i}\left(q_{i} ; \underline{q}_{-i}(n-1)\right) \quad \forall i .
$$

In a distributed system, ${ }^{\mathrm{h}}$ the corresponding continuoustime Jacobi iteration approximation is

$$
\dot{q}_{i}(t)=\frac{\partial V_{i}}{\partial q_{i}}(\underline{q}(t)) \quad \forall i
$$

and is motivated when players take small steps toward their currently optimal response, i.e., better-response dynamics [41]. That is to say, for positive step-size, $\varepsilon \ll 1$ (5) approximates the discrete-time better-response dynamics, 


$$
q_{i}(n)=q_{i}(n-1)+\varepsilon \frac{\partial V_{i}}{\partial q_{i}}(\underline{q}(n)) \quad \forall i,
$$

which is a kind of distributed gradient ascent. The Jacobi iteration is also motivated by the desire to take small steps to avoid regions of attraction of undesirable boundary NEPs, particularly those corresponding to the capture strategy $\left(q_{i}=1\right.$ for some $\left.i\right)$. Note that when more than one player selects this strategy, the result is a bad outcome for the game chicken or a deadlocked 'tragedy of the commons.' Additionally, the players avoid the opt-out strategy $\left(q_{i}=0\right.$ for some $\left.i\right)$. In summary, (6) represents a repeated game in which players adjust their transmission parameters $q_{i}$ to (locally) maximize their net utility $V_{i}$.

To find conditions on the parameters of net utilities (1) for local stability of the equilibria, we can apply the Hartman-Grobman theorem [42] to (5), i.e., to check that the Jacobian is negative definite. The following proposition uses the conditions of [43] for stability (and uniqueness) for a special case.

Proposition 3.2. In the case where players have the same parameters $C$ and $A$, the symmetric NEP $q^{*} \underline{1}$ is locally asymptotically stable under the dynamics in (5) when the normalized parameters satisfy

$$
C>2(N-1) A \text {. }
$$

Proof. By [43], the result follows if the symmetric $N \times N$ matrix $H(\underline{q})$ is negative definite, where

$$
H_{i j}=\frac{\partial^{2} V_{i}}{\partial q_{i} \partial q_{j}}+\frac{\partial^{2} V_{j}}{\partial q_{j} \partial q_{i}} .
$$

First note that for all $i$,

$$
H_{i i}(\underline{q})=-\frac{C}{q_{i}^{2}}<-C .
$$

For $l \neq i$,

$$
\begin{aligned}
\frac{\partial^{2} V_{i}}{\partial q_{i} \partial q_{l}}= & \frac{\partial}{\partial q_{l}}\left(\frac{C}{q_{i}}-A \alpha\left(\underline{q}_{-i}\right) \frac{1}{N-1} \sum_{j \neq i} q_{j} \prod_{k \neq i, j}\left(1-q_{k}\right)\right) \\
= & A \prod_{j \neq i, l}\left(1-q_{j}\right) \frac{1}{N-1} \sum_{j \neq i} q_{j} \prod_{k \neq i, j}\left(1-q_{k}\right) \\
& +A \alpha\left(\underline{q}_{-i}\right) \frac{1}{N-1}\left(\sum_{j \neq i, l} q_{j} \prod_{k \neq i, j, l}\left(1-q_{k}\right)\right. \\
& \left.-\prod_{k \neq i, l}\left(1-q_{k}\right)\right) .
\end{aligned}
$$

Now because $0<q_{i}<1$ for all $i$ and the triangle inequality,

$$
\left|H_{i j}(\underline{q})\right| \leq 2 A \quad \forall j \neq i \text {. }
$$

So, by the Gershgorin circle (disc) theorem (see p. 344 of [44]), all of $H(q)$ 's eigenvalues are less than $-C+(N-$ 1) $2 A$. So, if (7) holds, then all the eigenvalues of $H(q)$ are negative, and so $H(\underline{q})$ is negative definite.

\subsection{The marginal effect of altruism}

In this section, we will write $q^{*}$ (of the symmetric NEP $q^{*} \underline{1}$ in symmetric users case) as a function of the normalized altruism parameter $a:=A / M, q^{*}(a)$. Note that $q^{*}(0)=$ $c:=C / M$.

Recall that the total throughput for slotted ALOHA, $N c(1-c)^{N-1}$, is maximal when $c=1 / N$. The maximum total throughput is $(1-1 / N)^{N-1} \approx \mathrm{e}^{-1}$ for large $N$, i.e., the maximum throughput per player is $1 /(\mathrm{Ne})$ in this cooperative setting without networking costs.

So, if $c>1 / N$, i.e., total throughput is less than $\mathrm{e}^{-1}$ because of excessive demand (overloaded system), then a marginal increase in altruism from $0(0<a \ll 1)$ will cause a marginal decrease in $q^{*} \downarrow 1 / N$, resulting in an increase in throughput per user $\gamma \uparrow 1 /(\mathrm{Ne})$. Also, if $c<1 / N$, i.e., total throughput is less than $\mathrm{e}^{-1}$ because of a lack of demand (an underloaded system), then a marginal increase in altruism from 0 will again cause a marginal decrease in $q^{*}$, but here resulting in a decrease in throughput $\gamma$ (further away from the optimum $\mathrm{e}^{-1}$ ). See Section 5.4.

\section{Closely related model variations}

\subsection{Alternative altruism terms}

Obviously, different variations of our altruism parameter are possible, e.g., instead of the product of channel idleness and the mean throughput of other players, we could have considered the sum. We will herein consider the dynamic product form described above and its static version with no idleness term.

\subsection{Throughput-based costs}

In [22] we considered throughput-based costs with a static altruism parameter and with utility proportional to throughput. Instead of (1), for throughput-based costs with dynamic altruism and utility being a concave (log) function of throughput, we can model the net utility as

$$
\tilde{V}_{i}(\underline{q})=C_{i} \log \left(\gamma_{i}(\underline{q})\right)+A_{i} \alpha_{i}\left(\underline{q}_{-i}\right) \bar{\gamma}_{-i}(\underline{q})-M_{i} \gamma_{i}(\underline{q}) .
$$

Proposition 3.1 can easily be adapted for power-based costs. Instead of (3), the first-order necessary condition for a symmetric Nash equilibrium $q \underline{1}$ under throughputbased cost is

$$
\tilde{f}(q):=a q^{2}(1-q)^{2 N-3}+q(1-q)^{N-1}-c=0 .
$$

All solutions $q$ for (9) correspond to NEPs $q \underline{1}$ because $\partial^{2} \tilde{V}_{i}(\underline{q}) / \partial q_{i}^{2}=-C_{i} / q_{i}^{2}<0$ for all $i, \underline{q}$ (as for power-based cost). Note that $\tilde{f}(0)=\tilde{f}(1)=-c<0$, so we cannot 
simply use the intermediate value theorem as we did for Proposition 3.1 to establish existence of a symmetric Nash equilibrium when $c<1$. Here, existence requires

$$
\max _{0<q<1} \tilde{f}(q) \geq 0,
$$

a condition on $N, c, a$.

Note that if the inequality in (10) strictly holds, then there will be an even number of symmetric NEPs, again by the intermediate value theorem. If the maximum equals 0 , then there may be a unique symmetric NEP.

\subsection{Proportional throughput utility}

Suppose that utility is simply proportional to throughput and cost is power based, i.e., the net utility is

$$
\hat{V}_{i}(\underline{q})=C_{i} \gamma_{i}(\underline{q})+A_{i} \alpha_{i}\left(\underline{q}_{-i}\right) \bar{\gamma}_{-i}(\underline{q})-M_{i} q_{i}
$$

Note that the net utility $\hat{V}_{i}$ is linear in $q_{i}$ (this would also be the case if throughput-based costs were involved). This normally leads to candidate 'bang-bang' Nash equilibrium play actions, $q_{i} \in\{0,1\}$ for all players $i$; i.e., the players are either out of the game $\left(q_{i}=0\right.$ if $\left.\partial \hat{V}_{i} / \partial q_{i}<0\right)$ or are all in $\left(q_{i}=1\right.$ if $\left.\partial \hat{V}_{i} / \partial q_{i}>0\right)$. Note that the latter play action, potentially leading to the deadlock of tragedy of the commons, is not an equilibrium here because if $q_{j}=1$ then $\partial \hat{V}_{i} / \partial q_{i}=-M<0$ for all $i \neq j$.

It turns out that for this case, there is a symmetric interior equilibrium $q \underline{1}$ for the identical players case with $0<$ $q<1$, i.e., where

$$
\begin{aligned}
\hat{f}(q) & :=\frac{\partial \hat{V}_{i}}{\partial q_{i}}(q \underline{1}) \\
& =c(1-q)^{N-1}-a q(1-q)^{2 N-3}-1=0 .
\end{aligned}
$$

If $c>1, \hat{f}(0)=c-1>0$ and $\hat{f}(1)=-1<0$ and so there is a solution to $\hat{f}(q)=0$ for $0<q<1$ by the intermediate value theorem. It should be noted, however, that such an interior Nash equilibrium $q \underline{1}$ is not stable, i.e., it is a saddle point in the domain $[0,1]^{N}$.

\subsection{Heterogeneous players}

Asynchronous players were considered previously in [7] using the ideas from $[45,46]$. A very similar approach can be used to extend the results herein to account for the effects of asynchronous play. Numerical results for cases of heterogeneous players, including the special case of players with different play rates that are otherwise identical, are given in Section 6.

\section{Numerical studies for identical players at Nash equilibrium}

\subsection{Power-based costs}

For normalized utility parameter $c=0.5$ and normalized altruism parameter $a=1$, Figure 1 is a plot of $f$ in (3); i.e., for power-based costs and for $N=2,3,5,10$ players. The root at $q=0.4$ corresponds to $N=2$ (i.e., corresponding to NEP $q 1$ ) and, as the first term of $f$ becomes negligible, the root at $\approx 0.5$ corresponds to the $N>2$ cases. For $c=$ 0.5 and $N=5$, Figure 2 is a plot of $f$ for $a=0.1,1,10,100$. Note that $a=100$ corresponds to the larger curve which has a smaller root $q$, i.e., under 'excessive' altruism the NEP $q \rightarrow 0$.

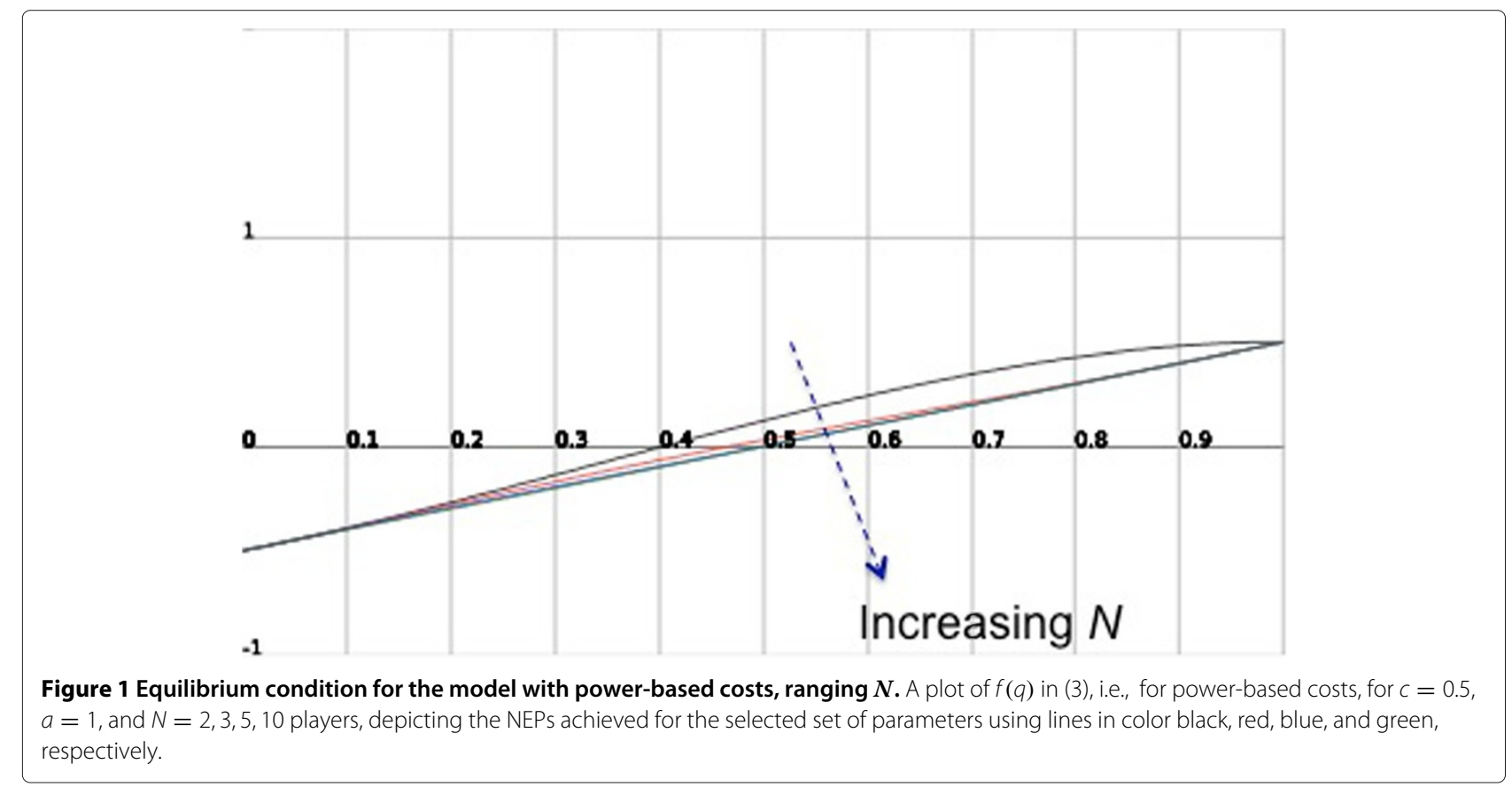




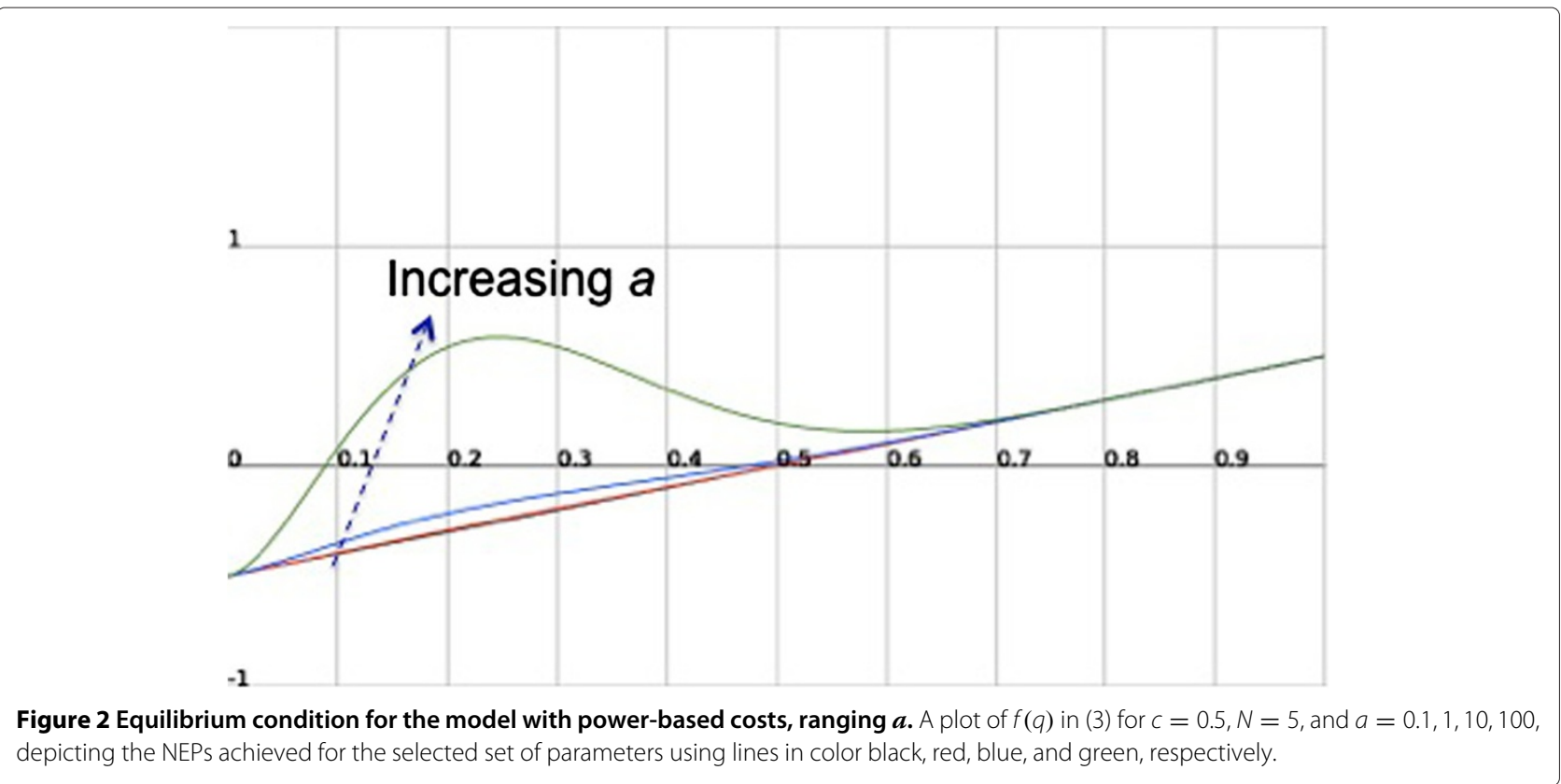

\subsection{Throughput-based costs}

Using the same parameter cases as those for power-based costs, Figure 3 is a plot of $\tilde{f}$ in (9) for $c=0.5, a=1$, and $N=2,3,5,10$. Figure 4 is a plot of $\tilde{f}$ in (9) again for $c=0.5, N=5$, and $a=0.1,1,10,100$. The larger curve, corresponding to $a=100$, has two zero-crossings $q$ at approximately 0.1 and 0.4 , i.e., has two different symmetric NEPs $q \underline{1}$. The other parameter sets do not possess an interior NEP, a situation which will be remedied if we reduce the utility $c$ from 0.5 to 0 , that is, increasing $\tilde{f}$.

\subsection{Throughput proportional utilities and costs}

Figure 5 is a plot of $\hat{f}$ in (12) for $c=2, a=1$, and $N=2,3,5,10$. Figure 6 is a plot of $\hat{f}$ for $c=2, N=5$, and $a=0.1,1,10,100$. Following intuition, the lower curves (and lower roots, NEPs) correspond to the larger $N$ (larger congestion leading to lower throughput) or larger

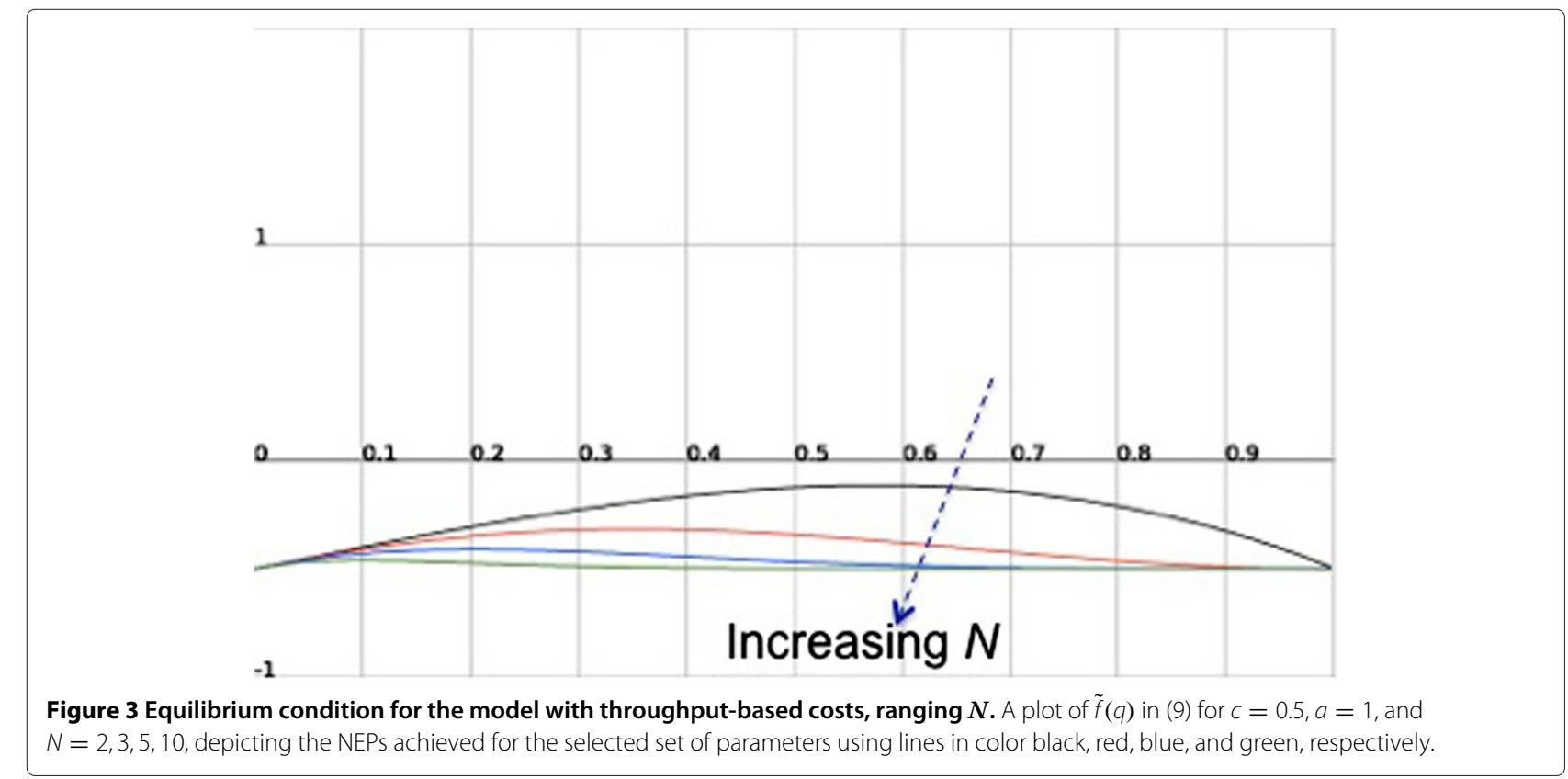




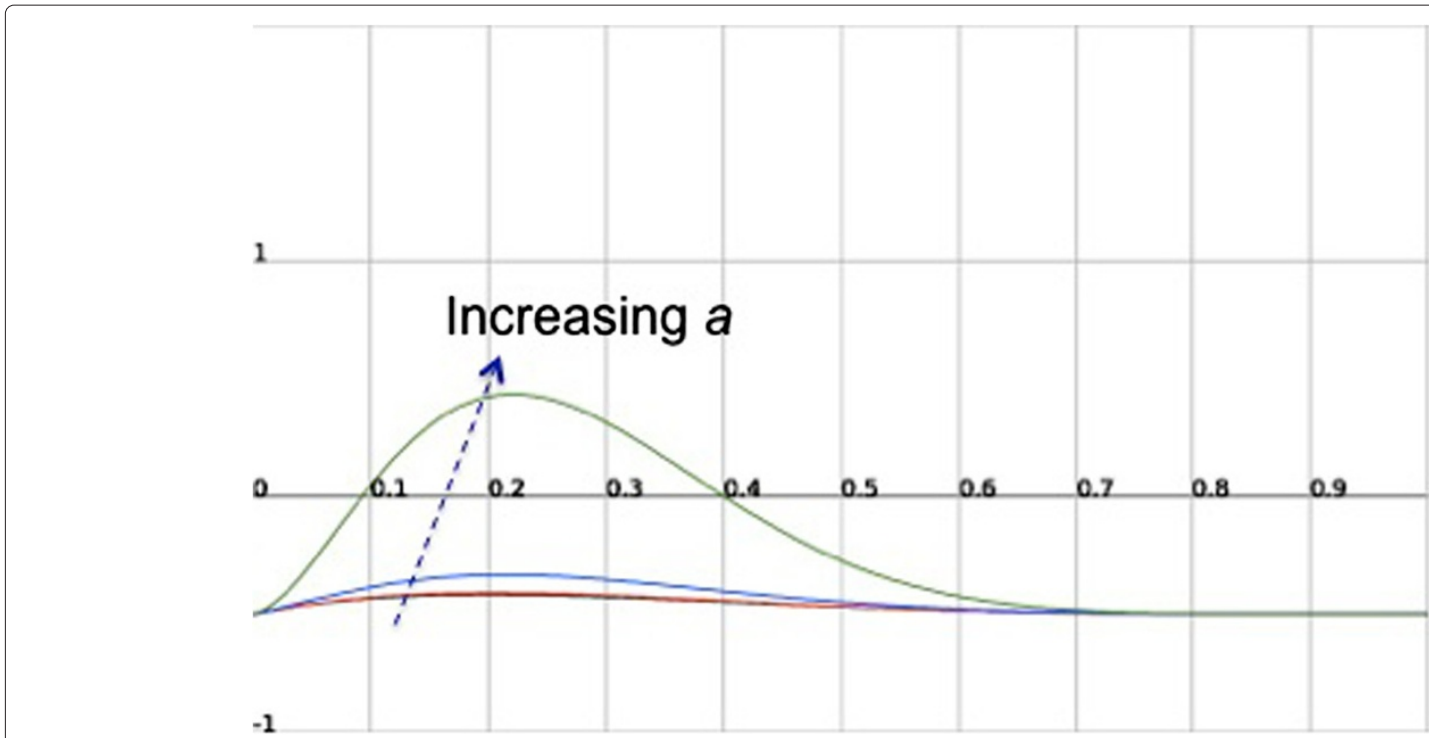

Figure 4 Equilibrium condition for the model with throughput-based costs, ranging $\boldsymbol{a}$. A plot of $\tilde{f}(q)$ in (9) for $c=0.5, N=5$, and $a=0.1,1,10,100$, depicting the NEPs achieved for the selected set of parameters using lines in color black, red, blue, and green, respectively.

$a$ (greater altruism again leading to lower throughput), in a monotonic fashion when all other parameters are fixed.

5.4 An example comparing altruism and non-cooperation In this section, we compare the Nash equilibria under altruistic player action with equilibria in purely non cooperative scenarios. For all scenarios, we considered the case of power-based costs, log-utility of throughput, normalized utility parameter $c=0.5$, and identical users. For the purely non-cooperative scenario, i.e., $a=0$, the symmetric Nash equilibrium $q=c=0.5$ is simply obtained by solving (3). For the scenarios with altruism, the normalized altruism parameter was taken to be $a=20$. Recall that for static altruism, $\alpha \equiv 1$. At Nash equilibrium $q^{*}=q^{*} \underline{1}$, the throughput $\left(\gamma^{*}=q^{*}\left(1-q^{*}\right)^{N-1}\right)$ and utility (1) performance per user are given in the following table, in decreasing order of throughput. For a third scenario, assume that the players cooperated by rule to

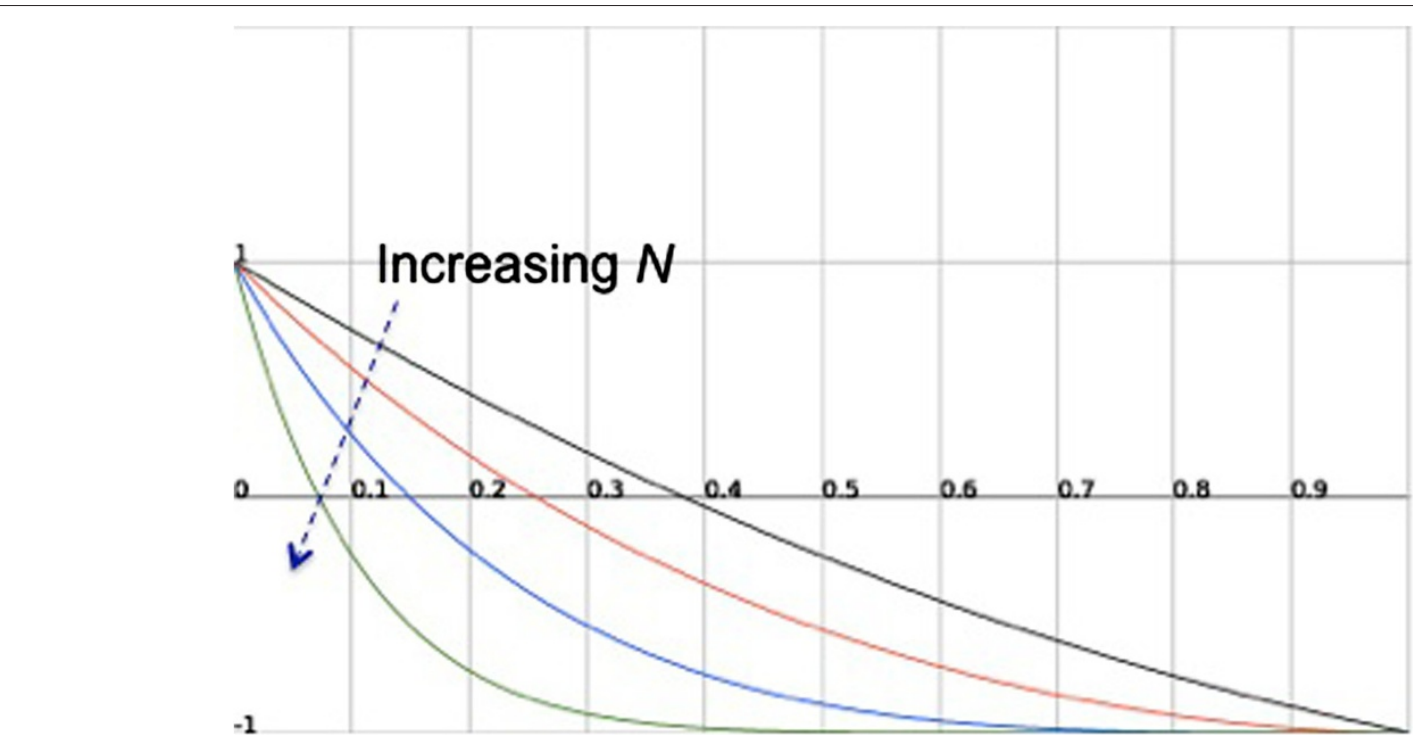

Figure 5 Equilibrium condition for the model with throughput proportional utility and power-based cost, ranging $\boldsymbol{N}$. A plot of $\hat{f}(q)$ in (12) for $c=2, a=1$, and $N=2,3,5,10$, depicting the NEPs achieved for the selected set of parameters using lines in color black, red, blue, and green, respectively. 


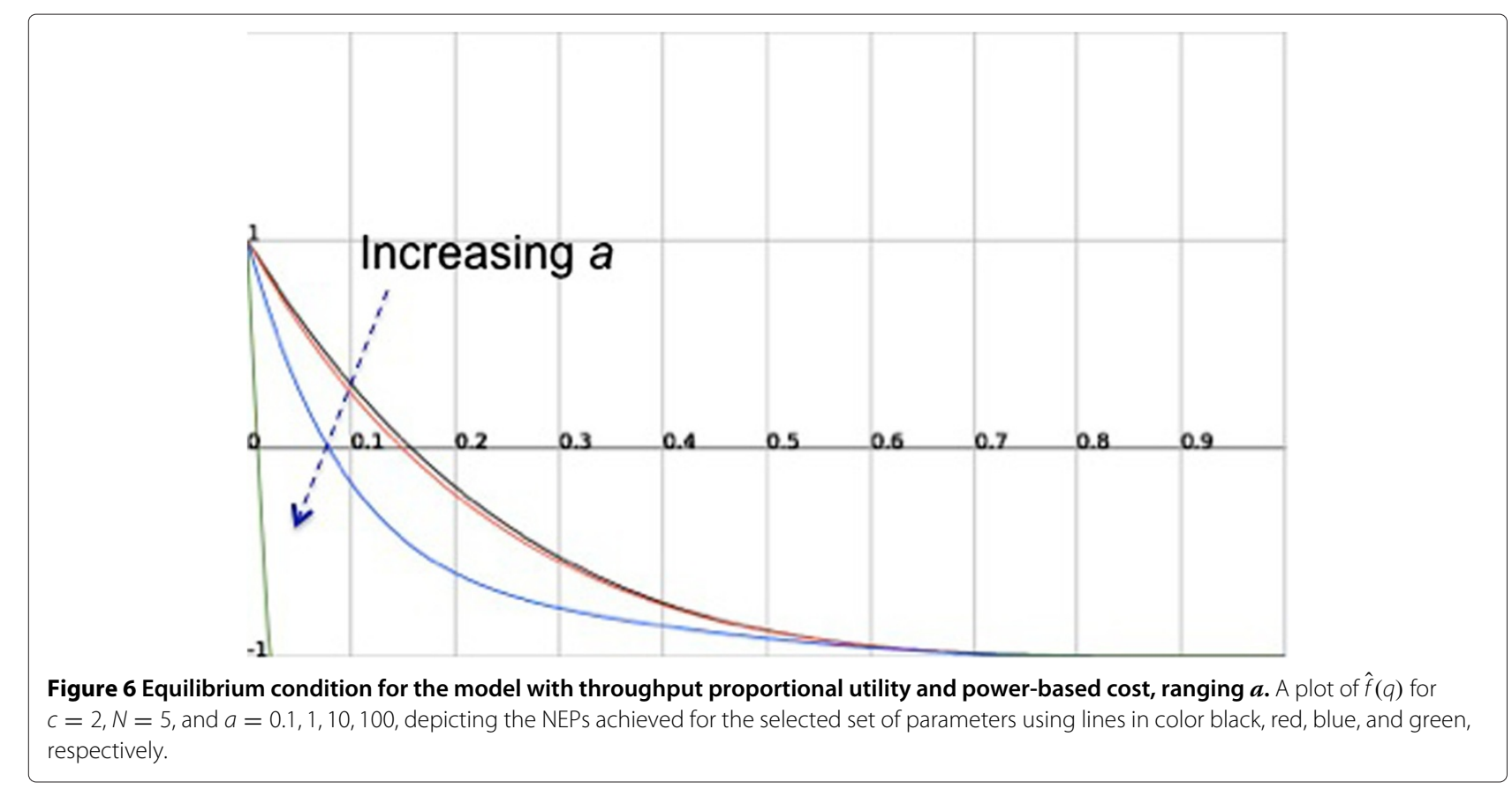

maximize social welfare ${ }^{\mathrm{i}} W=\sum_{i} V_{i}$, where $V_{i}$ given by (3) with $a=0$; the first-order condition for the symmetric Nash equilibrium $q \underline{1}, \partial W / \partial q_{i}=0$, is equivalent to

$$
q^{2}-(N c+1) q+c=0
$$

As depicted in Table 2, our dynamic altruism model does not perform well (in terms of mean throughput per user at Nash equilibrium) when contention is high, as it is the case for $N=8$, under the assumed parameters.

If there is little demand, the idle time will be high and the throughput low, so the altruism term may or may not be high. If there is congestion, the idle time will be low and

Table 2 Numerical comparison

\begin{tabular}{llll}
\hline Scenario & $\boldsymbol{q}^{*}$ & $\boldsymbol{\gamma}^{*}$ & $\boldsymbol{V}^{*} / \mathbf{M}$ \\
\hline$N=4$ & & & \\
Dynamic altruism & 0.22 & 0.1044 & -0.36 \\
Cooperative & 0.18 & 0.0972 & -1.34 \\
Static altruism & 0.16 & 0.0935 & 0.53 \\
Non-cooperative & 0.50 & 0.0625 & -1.89 \\
$N=8$ & & & \\
Cooperative & 0.10 & 0.0478 & -1.62 \\
Static altruism & 0.28 & 0.0277 & -1.52 \\
Dynamic altruism & 0.50 & 0.0039 & -3.27 \\
Non-cooperative & 0.50 & 0.0039 & -3.27 \\
\hline
\end{tabular}

The results of a numerical example comparing altruism and non-cooperation (see section 5.4). the throughput will also be low (the latter due to interference), so the altruism term will be low. Then the players tend to be non-cooperative in the congested state (when $N$ is high). Considering the static altruism model, for which channel idle time is not a factor, one expects that the players tend to be non-cooperative in the congested state too (throughput is low due to interference), but the altruism term will be higher since the alpha (idle time) term is not involved, so the tendency to be non-cooperative will be for a higher $N$ under static altruism than under our dynamic altruism - as is the case for $N=8$.

For $N=4$, dynamic altruism does better owing to both higher channel idle time and higher total throughput. In this low-contention case, the dynamic altruism term is smaller than in the static case (owing to the idle-time component $\alpha$ ) so users will value their own throughput utility more (and they can achieve more throughput owing to less contention), which is collectively re-enforced by the throughput component of the dynamic altruism term. Furthermore, for this low-contention case, the cooperative strategy has simply 'met its demands' (maximized utility minus cost), and under power-based costs, increasing transmission probability has a great reduction on cost components of the net utility terms and again boosts the altruism components of the utility of other players.

\section{Numerical studies with player diversity}

\subsection{Players with different altruism parameters}

Consider the game with power-based costs. In this section, we consider players with different normalized altruism parameters $a$ for $N=3$, which are otherwise 
Table 3 The role of $a$

\begin{tabular}{cccc}
\hline $\boldsymbol{a}_{\mathbf{1}}$ & $\boldsymbol{q}_{1}^{*}, \boldsymbol{q}_{2}^{*}=\boldsymbol{q}_{3}^{*}$ & $\boldsymbol{\gamma}_{1}^{*}, \boldsymbol{\gamma}_{2}^{*}=\boldsymbol{\gamma}_{3}^{*}$ & $\mathrm{i} \boldsymbol{V}_{1}^{*}, \boldsymbol{V}_{\mathbf{2}}^{*}=\boldsymbol{V}_{3}^{*}$ \\
\hline 30 & $0.15,0.10$ & $0.13,0.074$ & $0.754,2.37$ \\
40 & $0.12,0.10$ & $0.10,0.080$ & $1.40,2.24$ \\
50 & $0.10,0.10$ & $0.083,0.083$ & $2.10,2.10$ \\
60 & $0.091,0.11$ & $0.073,0.087$ & $2.79,1.83$ \\
70 & $0.079,0.11$ & $0.063,0.090$ & $3.56,1.82$ \\
\hline
\end{tabular}

The role of $a$ in the NEP $q^{*}$ and corresponding throughputs $\gamma^{*}$ and utilities $V^{*}$ per user.

identical players with normalized parameter $c=0.5$ associated with power-based cost. Specifically, the first player has $a_{1}$ ranging from 30 to 70 , while the other two players both have $a=50$. Note that changing $a$ in this manner will result in changes in the NEP $q^{*}$ and corresponding throughputs $\gamma^{*}$ (and utilities $V^{*}$ per user, as shown in Table 3).

Following intuition, increased altruism $\left(a_{1}>50\right)$ by player 1 resulted in lower throughput for him and higher throughput for the other two players. Similarly, decreased altruism by player $1\left(a_{1}<50\right)$ resulted in higher throughput for him and lower throughput for the other players.

\subsection{Sizes of regions of attractions under different play rates}

In this section, we study how the volume of the regions of attractions of different equilibria is sensitive to players adopting different play rates, while retaining our assumption of fictitious/quasi-stationary play. Consider the case of $N=3$ players two of whom have the same play rate while the other adopts a play rate that is a multiple, $r$, of the other two. We consider the case of throughput-based costs as in Figure 4. That is to say,

$$
\dot{q}_{i}(t)=r_{i} \frac{\partial \tilde{V}_{i}}{\partial q_{i}}(\underline{q}(t)) \quad \forall i
$$

where $r_{i}=r$ for player $i=1$; otherwise, $r_{i}=1$ and $\tilde{V}$ is given in Section 4.2. Numerically simulating (13) from different initial points chosen from a grid in the hypercube $[0,1]^{3}$, we counted the number of initial points converging to a given NEP so as to estimate the volume of its region of attraction. Note that the introduction of such play-rate parameters $r_{i}$ does not change the position of the NEPs. Using normalized parameters $a=50$ and $c=0.5$, the function $\tilde{f}$ whose roots are the NEPs is depicted in Figure 7. As the results presented in Table 4 demonstrate, the region of attraction is very sensitive to $r$ in the range 0.1 to 10 .

Again following intuition, a lower $r$ effectively corresponds to a reluctance to be altruistic and thereby results in a smaller domain of attraction for the more altruistic Pareto (higher $\tilde{V}$ ) equilibrium (0.1) $\underline{1}$.

\subsection{Players with different transmission priorities}

Now consider the case where the factors $C_{i}$ in (1) are different. Equivalently, assume the factors $r_{i}$ affect only the throughput utility rather than the whole net utility as they do in (13). Taking the cases of such factors as in the previous Subsection 6.2 again leads to intuitively consistent results as above: users with higher priority will achieve more throughput at Nash equilibrium.

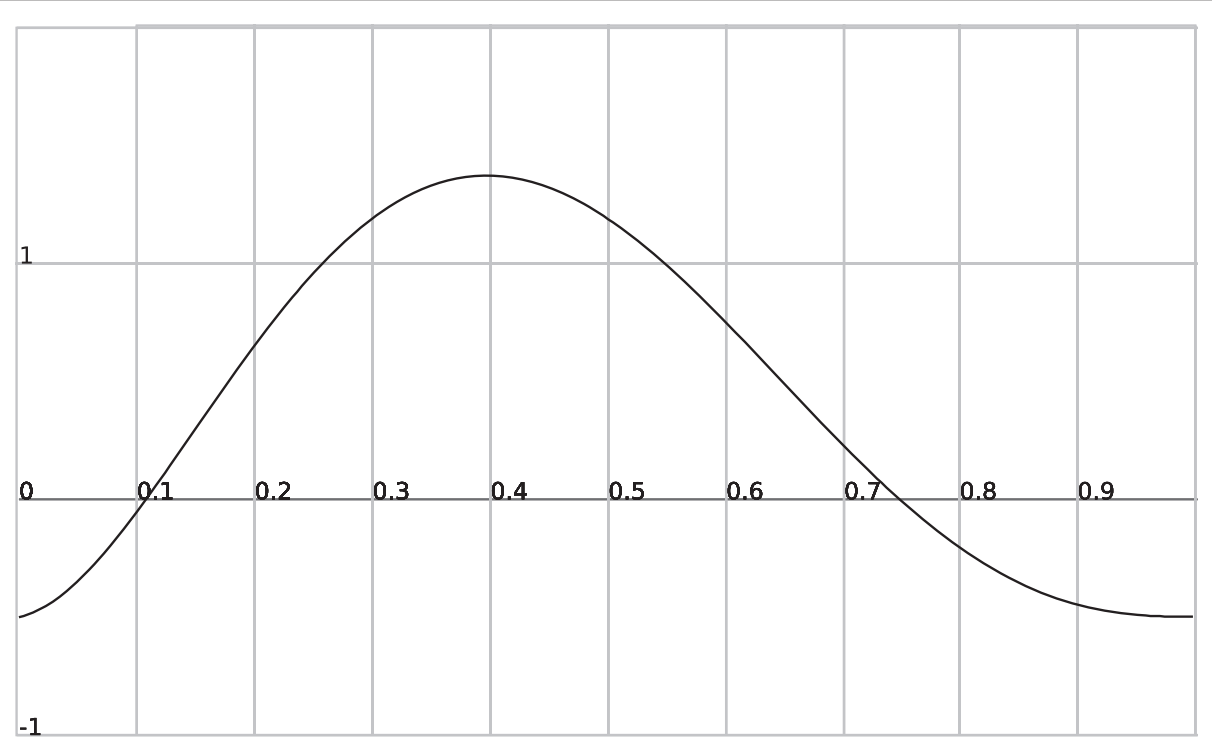

Figure 7 Equilibrium condition of the model with power-based costs for $\boldsymbol{N}=\mathbf{3}$. A plot of the function $\tilde{f}(q)$ whose roots are the NEPs, using normalized parameters $a=50$ and $c=0.5$. 
Table 4 The role of $r$

\begin{tabular}{ccc}
\hline Volume & NEP $=(\mathbf{0 . 1}) \underline{\mathbf{1}}$ & $\mathbf{N E P}=\mathbf{( 0 . 7 5 )} \boldsymbol{\underline { 1 }}$ \\
\hline$r=0.1$ & 0.502 & 0.498 \\
$r=0.25$ & 0.507 & 0.493 \\
$r=1$ & 0.556 & 0.444 \\
$r=4$ & 0.839 & 0.161 \\
$r=10$ & 0.841 & 0.159 \\
$\gamma:$ & 0.081 & 0.047 \\
$\tilde{V}:$ & 1.94 & -1.43 \\
\hline
\end{tabular}

The role of $r$ in the NEP $q^{*}$.

\section{Conclusions}

In this paper, we extended a non-cooperative game framework for information-limited MAC of a LAN by adding an altruism term that depended on both the mean throughput of the other players and the mean channel idle time. The cases of heterogeneous or homogeneous users, and of power or throughput-based costs, were considered for a quasi-stationary model of the game. A numerical study compares the per-user throughput under dynamic and static altruism with that of purely non-cooperative dynamics, and demonstrates the advantage of altruism under moderate levels of congestion (number of players) in the homogeneous player setting and for a heterogeneous user scenario. Our numerical studies produced intuitive results which means that our model is selfconsistent and could form the basis for more sophisticated extensions. However, our dynamic altruism term is sensitive to the use of the mean idle time as a measure of the current level of altruism in the system, which could lead to wrong interpretations in certain scenarios (e.g., when demand is low or when congestion is due to a high number of users in the channel). In more advanced versions of our model, we will include the number of competing users, $N$, in the term expressing the current level of altruism in the system in order to avoid such misinterpretations.

In the future, we will also consider a mixed scenario of of three types of players:

- Those that follow an original protocol that enacts distributed/information-limited cooperation (by rule) for flow and congestion control

- Altruistic but pragmatic (second defectors) who will defect to avoid starvation, and

- Selfish (first defectors), who will cooperate only to avoid starvation.

Note that both types of defectors can engage in an evolutionary cycle ${ }^{j}$ of tactical transitions as they assess the social communication norms in the LAN among users who are active presently and in the recent past, classifying the active users into the above three categories in particular. One can ask what distributed congestion and flow control protocol can best deal with defectors of both types ${ }^{k}$ ? Given that players will be intermittently active or may be active with communication of differing degrees of priority, can an altruistic framework (possibly with an evolutionary 'wrapper') be designed to effectively conduct priority scheduling in this LAN context? A challenge here is dealing with the greedy user who declares all of their communication as high priority. Again, our ultimate aim is to achieve fair and efficient throughputs for by rule cooperators and altruistic defectors alike, while not starving-out/shunning the deemed selfish defectors.

\section{Endnotes}

a In this paper, we use the terms user, player, participant and node interchangeably.

b I.e., without steady-state estimates of certain quantities.

c Such estimates are feasible in our application context of a CSMA local area (broadcast) network with a relatively small population of active participants, but is not possible for many other networking contexts, e.g., the example of TCP congestion control mentioned above. So, it would be difficult to obtain estimates of 'social norms' to form the basis of 'rational' altruism for TCP.

d See http://dictionary.reference.com/browse/altruism

e Power-based costs are borne whether or not the transmission is successful.

${ }^{\mathrm{f}}$ Obviously, we could have combined $\alpha_{i}$ and $\bar{\gamma}_{-i}$ in different ways, instead of a product form, to form an altruism modifier for the net utility of player $i, c f$. the next section for other model variations.

g I.e., not including the stable boundary deadlock equilibrium at $\underline{q}=\underline{1}$.

h $c f$. Section 4.4 for a discussion of asynchronous play.

i This is similar to the classical slotted ALOHA example where all (identical) players choose a common $q=1 / N$ to maximize total (and individual) throughputs(without considering costs).

j Possibly at a slow time scale of human response.

k At a (faster) time scale of machine response.

Competing interests

The authors declare that they have no competing interests.

\section{Acknowledgements}

GK was supported by NSF CISE grants 0524202 and 0915928 by a Cisco Systems URP gift; PA was supported by EINS, the Network of Excellence in Internet Science EC's FP7 grant 288021. YJ was supported by NRF-2013R1A1A3A04007104. Elements of this paper were presented at the IEEE CDC, Atlanta, 2010 and AdHocNets, Paris, 2012 conferences.

\section{Author details}

${ }^{1}$ ETH Zurich, Zurich, Switzerland. ${ }^{2}$ UPMC Sorbonne Universités, Paris, France. ${ }^{3}$ The Pennsylvania State University, University Park, PA, USA. ${ }^{4}$ KAIST, Daejeon, South Korea. 
Received: 22 November 2012 Accepted: 10 July 2013

\section{Published: 1 August 2013}

\section{References}

1. M Cagalj, S Ganeriwal, I Aad, J Hubaux, On selfish behavior in CSMA/CA networks, in Proceedings of the IEEE INFOCOM, (Miami, 13-17 March 2005)

2. M Raya, I Aad, JP Hubaux, AE Fawal, DOMINO: detecting MAC layer greedy behavior in IEEE 802.11 hotspots. IEEE Trans. Mobile Comput. 5(12), 1691-1705 (2006)

3. A Akella, S Seshan, R Karp, S Shenker, C Papadimitriou, Selfish behavior and stability of the Internet: A game-theoretic analysis of TCP, in Proceedings of ACM SIGCOMM, (Pittsburgh, pp. 19-23 August 2002

4. $Y$ Jin, $G$ Kesidis, A pricing strategy for an ALOHA network of heterogeneous users with inelastic bandwidth requirements, in Proceedings of the CISS, (Princeton, 20-22 March 2002)

5. T Alpcan, T Basar, R Srikant, E Altman, CDMA uplink power control as a noncooperative game. Wireless Netw. 8, 659-670 (2002)

6. SWicker, A Mackenzie, Stability of multipacket slotted Aloha with selfish users and perfect information, in Proceedings of IEEE INFOCOM, (San Francisco, CA, 30 March-3 April 2003)

7. Y Jin, G Kesidis, Dynamics of usage-priced communication networks: the case of a single bottleneck resource. IEEE/ACM Trans. Network. 13, 1041-1053 (2005)

8. F Meshkati, M Chiang, H Poor, S Schwartz, A game-theoretic approach to energy-efficient power control in Multicarrier CDMA systems. IEEE JSAC. 24(6), 1115-1129 (2006)

9. J Lee, M Chiang, R Calderbank, Utility-optimal random-access protocol. IEEE Trans. Wireless Commun. 6(7), 2741-2751 (2007)

10. C Long, Q Zhang, B Li, H Yang, X Guan, Non-cooperative power control for wireless ad hoc networks with repeated games. IEEE J. Selected Areas Commun. 25(6), 1101-1112 (2007)

11. $Y$ Jin, $G$ Kesidis, Distributed contention window control for selfish users in IEEE 802.11 wireless LANs. IEEE JSAC Spec. Issue. Non-cooperative Netw. 25, 1113-1123 (2007)

12. T Cui, L Chen, S Low, A game-theoretic framework for medium access control. IEEE J. Selected Areas Commun. 26(7), 1116-1127 (2008)

13. R Ma, V Misra, D Rubenstein, An analysis of generalized slotted-aloha protocols. IEEE/ACM Trans. Netw. 17(3), 936-949 (2009)

14. J Weibull, Evolutionary Game Theory (MIT Press, Cambridge, MA, 1995)

15. N Nowak, Five rules for the evolution of cooperation. Science. $\mathbf{3 1 4}$ 1560-1563 (2006)

16. C Camerer, G Loewenstein, ed. by C Camerer, G Loewenstein, and M Rabin, Behavioral economics: past, present, future, in Advances in Behavioral Economics (Princeton Univ. Press, Princeton, 2003)

17. M Huang, P Caines, R Malhame, Social dynamics in mean field LQG control: egoistic and altruistic agents, in IEEE Conference on Decision and Control (CDC) (IEEE, Atlanta, Georgia, 15-17 December 2010)

18. K Avrachenkov, J Elias, F Martignon, G Neglia, L Petrosyan, A Nash bargaining solution for cooperative network formation games, Networking 2011 Lecture Notes in Computer Science. 6640, 307-318

19. P Hui, KXu, V Li, J Crowcroft, V Latora, P Lio, Selfishness, altruism and message spreading in mobile social networks, in Proceedings of the First IEEE International Workshop on Network Science For Communication Networks (NetSciCom), (Rio de Janeiro, 24 April 2009)

20. A Azad, E Altman, R Elazouzi, From altruism to non-cooperation in routing Games, in Proceedings of Networking and Electronic Commerce Research Conference (NAEC) (ATSMA, Dallas, 8-11 October 2009)

21. Y Sharma, D Williamson, Stackelberg thresholds in network routing games or the value of altruism. Games Econ. Behav. 67, 174-190 (2009)

22. G Kesidis, Y Jin, A Amar, E Altman, Stable Nash equilibria of LAN medium access games under symmetric, socially altruistic behavior, in Proceedings of IEEE CDC 2010. [Technical report number arXiv:1003.5324 available at http://arxiv.org]

23. M Heusse, F Rousseau, R Guillier, A Dula, Idle sense: an optimal access method for high throughput and fairness in rate diverse wireless $L A N s$, in Proceedings of ACM SIGCOMM. (ACM, New York, 2005), pp. 121-132
24. J Ledyard, A Roth, ed. by J Kagel, Public goods: a survey of experimental research, in Handbook of Experimental Economics (Princeton University Press, Princeton, 1995)

25. C Camerer, E Fehr, ed. by J Henrich, R Boyd, S Bowles, C Camerer, E Fehr, and $\mathrm{H}$ Gintis, Measuring social norms and preferences using experimental games: a guide for social scientists, in Foundations of Human Sociality: Economic Experiments and Ethnographic Evidence from Fifteen Small-Scale Societies (Oxford University Press, Oxford, 2004)

26. SD Levitt, JA List, What do laboratory experiments measuring social preferences reveal about the real world? J. Econ Perspect. 21(2), 153-174 (2007)

27. E Fehr, U Fischbacher, The nature of human altruism. Nature. $\mathbf{4 2 5}$ 785-791 (2003)

28. C Bicchieri, E Xiao, Do the right thing: but only if others do so. J. Behav. Decis. Mak. 22, 191-208 (2009)

29. M Dufwenberg, S Gächter, H Hennig-Schmidt, The framing of games and the psychology of play. Games Econ. Behav. 73(2), 459-478 (2011)

30. E Ostrom, Collective action and the evolution of social norms. J. Econ. Perspect. 14(3), 137-158 (2000)

31. C Bicchieri, The Grammar of Society: the Nature and Dynamics of Social Norms (Cambridge University Press, New York, 2006)

32. E Fehr, K Schmidt, A theory of fairness, competition, and cooperation. Q. J. Econ. 114(3), 817-868 (1999)

33. H Margolis, Cognition and Extended Rational Choice (Routledge, New York, 2007)

34. G Brown, ed. by T Koopmans, Iterative solutions of games with fictitious play, in Activity Analysis of Production and Allocation (Wiley:, New York, 1951)

35. Y Jin, G Kesidis, Equilibria of a noncooperative game for heterogeneous users of an ALOHA network. IEEE Commun. Lett. 6(7), 282-284 (2002)

36. Y Jin, G Kesidis, A channel-aware MAC protocol in an ALOHA network with selfish users. IEEE JSAC Spec. Issue on Game Theory Wireless Commun. 30, 128-137 (2012)

37. J Seade, The stability of Cournot revisited. J. Econ. Theory. 23, 15-27 (1980)

38. A Al-Nowaihi, P Levine, The stability of the Cournot oligopoly model: a reassessment. J. Econ. Theory. 35, 307-321 (1985)

39. A Zhang, Y Zhang, Stability of Nash equilibrium: the multiproduct case. J. Math. Econ. 26(4), 441-462 (1996)

40. I Menache, N Shimkin, Fixed-rate equilibrium in wireless collision channels, in Proceedings of the Network Control and Optimization (NET-COOP), (Avignon, 5-7 June 2007)

41. J Shamma, G Arslan, Dynamic fictitious play, dynamic gradient play, and distributed convergence to Nash equilibria. IEEE Trans. Auto. Contr. 50(3), 312-327 (2005)

42. L Perko, Differential Equations and Dynamical Systems (Springer-Verlag, New York, 2011)

43. J Rosen, Existence and uniqueness of equilibrium points for concave $\mathrm{N}$-person games. Econometrica. 33(3), 520-534 (1965)

44. R Horn, C Johnson, Matrix Analysis (Cambridge Univ. Press, Cambridge, 1988)

45. T Basar, G Olsder, Dynamic Noncooperative, Game Theory. Academic Press, 2nd edition (Society for Industrial and Applied Mathematics, Philadelphia, 1999)

46. D Bertsekas, J Tsitsiklis, Convergence rate and termination of asynchronous iterative algorithms, in Proceedings of the 3rd International Conference on Supercomputing, (Crete, 5-9 June 1989)

doi:10.1186/1687-1499-2013-202

Cite this article as: Antoniadis et al:: Distributed medium access control with conditionally altruistic users. EURASIP Journal on Wireless Communications and Networking 2013 2013:202. 